\title{
Single-cell genomic profiling of acute myeloid leukemia for clinical use: A pilot study
}

\author{
BENEDICT YAN ${ }^{1 *}$, YONGLI HU ${ }^{2 *}$, KENNETH H.K. BAN ${ }^{3}$, ZENIA TIANG $^{4,5}$, \\ CHRISTOPHER NG ${ }^{1}$, JOANNE LEE ${ }^{6}$, WILSON TAN ${ }^{4}$, LILY CHIU ${ }^{1}$, TIN WEE TAN ${ }^{7}$,

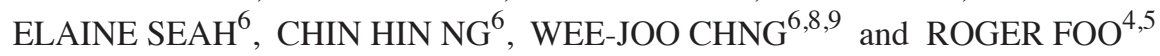 \\ ${ }^{1}$ Department of Laboratory Medicine, Molecular Diagnosis Centre, National University Health System, Singapore 119228; \\ ${ }^{2}$ Institute for Infocomm Research, Agency for Science, Technology and Research, Singapore 138632; \\ ${ }^{3}$ Department of Biochemistry, National University of Singapore, Singapore 117596; ${ }^{4}$ Genome Institute of Singapore, \\ Singapore 138672; ${ }^{5}$ Cardiovascular Research Institute, National University Health System; \\ ${ }^{6}$ Department of Hematology-Oncology, National University Cancer Institute, National University Health System, \\ Singapore 119228; ${ }^{7}$ National Supercomputing Centre, Singapore 138632; ${ }^{8}$ Cancer Science Institute, \\ National University of Singapore, Singapore 117599; ${ }^{9}$ Department of Medicine, Yong Loo Lin School \\ of Medicine, National University of Singapore, Singapore 117597, Republic of Singapore
}

Received July 11, 2015; Accepted November 21, 2016

DOI: $10.3892 / \mathrm{ol} .2017 .5669$

\begin{abstract}
Although bulk high-throughput genomic profiling studies have led to a significant increase in the understanding of cancer biology, there is increasing awareness that bulk profiling approaches do not completely elucidate tumor heterogeneity. Single-cell genomic profiling enables the distinction of tumor heterogeneity, and may improve clinical diagnosis through the identification and characterization of putative subclonal populations. In the present study, the challenges associated with a single-cell genomics profiling workflow for clinical diagnostics were investigated. Single-cell RNA-sequencing (RNA-seq) was performed on 20 cells from an acute myeloid leukemia bone marrow sample. Putative blasts were identified based on their gene expression profiles and principal component analysis was performed to identify outlier cells. Variant calling was performed on the single-cell RNA-seq data. The present pilot study demonstrates a proof of concept for clinical single-cell genomic profiling. The recognized limitations include significant stochastic RNA loss and the relatively low throughput of the current proposed platform. Although the results of the present study are promising, further technological advances and protocol
\end{abstract}

Correspondence to: Professor Roger Foo, Genome Institute of Singapore, 60 Biopolis Street, Singapore 138672, Republic of Singapore

E-mail: foosyr@gis.a-star.edu.sg

*Contributed equally

Key words: single cell, genomics, acute myeloid leukemia, transcriptomics, gene expression optimization are necessary for single-cell genomic profiling to be clinically viable.

\section{Introduction}

Bulk high-throughput genomic profiling studies have improved the understanding of cancer biology and facilitated the development of novel therapeutics. However, there is increasing awareness that bulk profiling approaches do not adequately produce information concerning tumor heterogeneity, an improved insight into which may facilitate the development of more effective therapeutic strategies (1).

Genomic profiling of individual single cells is currently technically available and recent reports of the highly parallel expression profiling of thousands of cells suggest that single-cell genomic profiling for clinical applications may become a reality $(2,3)$. Notably, single-cell profiling using flow cytometry for immunophenotyping is currently a routine hematological diagnostic assay (4). Single-cell genomic profiling is therefore, in theory, potentially of clinical utility in the diagnostic work-up of a hematological malignancy such as acute myeloid leukemia (AML).

AML is a malignant disease of abnormally differentiated cells of the hematopoietic system (5). It is a clonally complex disease that is characterized by the presence of multiple clonal populations in the primary cancer, any of which may evolve to result in relapse (6).

Single-cell genomic profiling enables the distinction of tumor heterogeneity, and may improve clinical diagnostics through the identification of putative subclonal populations and their respective drug sensitivity profiles (Fig. 1). In an attempt to develop a clinically relevant single-cell genomic profiling protocol, a pilot study of single-cell RNA-sequencing (RNA-seq) of an acute myeloid leukemia (AML) sample was performed. 


\section{Materials and methods}

Sample. An AML bone marrow sample, which was harvested in February 2011 from a 35-year-old female patient, was obtained from the archives of the Department of Hematology-Oncology (National University Hospital, Singapore). Ethical approval was obtained for the present study (Domain Specific Review Boards; National Healthcare Group, Singapore; ref. 2016/00547). Informed consent was obtained from the subject participating in the present study.

Flow cytometry. A total of 250,000 events were acquired for multiparametric analysis using a lyse-wash method on bone marrow cells (7). Blasts were identified using a cluster of differentiation (CD) 45/CD34/CD117/human leukocyte antigen-antigen D related (HLA-DR) combination (7).

Single-cell isolation for RNA-seq. A total of $5 \times 10^{6}$ cells were incubated with anti-CD45 antibody (Miltenyi Biotec, Inc., Cambridge, MA, USA; cat. no. 130-080-201; clone, 5B1) for $1 \mathrm{~h}$ at $4^{\circ} \mathrm{C}$ to stain white blood cells, while Hoechst 33342 (Thermo Fisher Scientific, Inc., Waltham, MA, USA; cat. no. H3570) was used to stain nuclei by adding the staining solution to the cells for $1 \mathrm{~h}$ at $4^{\circ} \mathrm{C}$. Cells were loaded at the optimal concentration $(250,000$ cells $/ \mathrm{ml}$, as recommended by the manufacturer) into the microfluidics chip.

Single cells were isolated into individual chambers using an integrated fluidic circuit (IFC) on the Automated Microfluidic C1 system (Fluidigm Corporation, San Francisco, CA, USA). Cells positive for CD45 and Hoechst were lysed, and RNA isolation and complementary DNA (cDNA) synthesis was performed using the SMART-Seq ${ }^{\circledR}$ v4 Ultra ${ }^{\circledR}$ Low Input RNA kit for Sequencing (Clontech Laboratories, Inc., Mountainview, CA, USA; cat. no. 634888) which was preamplified using a unique SMARTer II A oligonucleotide and template switch primer (both reagents being present in the SMARTer Ultra Low RNA kit; Clontech Laboratories, Inc.; cat. no. 634833), according to the manufacturer's protocol. The cDNA was harvested manually by retrieving $3.5 \mu 1$ of cDNA from the wells of the IFC for library preparation. Notably, only RNA strands with polyadenylated [poly(A)] tails were converted to cDNA and used for downstream processing.

Library preparation and next-generation sequencing. Using the coordinates from the imaging, 20 cells that stained positive for the leukocyte marker CD45 and had intact nuclei, as observed using the Hoechst stain, were selected. Library preparation was performed using the Nextera XT DNA Sample Preparation kit (Illumina, Inc., San Diego, CA, USA; cat. no. FC-131-1096), according to the manufacturer's protocol. The 20 libraries were processed individually, with each library being assigned a unique barcode for pooled multiplex sequencing using the Illumina HiSeq 2000 platform (Illumina, Inc.), according to the manufacturer's protocol. Paired-end 100 bp reads were generated for analysis.

Mapping and quantification of single-cell RNA-seq data. Paired-end FASTQ files were initially mapped to the reference human $\mathrm{Hg} 19$ transcriptome (ftp.ensembl.
Table I. Number of RNA-sequencing reads per cell.

\begin{tabular}{lc}
\hline Cell number & Number of reads, millions \\
\hline RHA100 & 6.3 \\
RHA101 & 9.9 \\
RHA102 & 9.4 \\
RHA103 & 5.1 \\
RHA104 & 4.7 \\
RHA105 & 8.2 \\
RHA106 & 11.4 \\
RHA107 & 7.4 \\
RHA108 & 7.8 \\
RHA109 & 9.2 \\
RHA110 & 5.5 \\
RHA111 & 5.2 \\
RHA112 & 5.2 \\
RHA113 & 5.9 \\
RHA114 & 4.5 \\
RHA115 & 11.4 \\
RHA116 & 9.3 \\
RHA117 & 6.8 \\
RHA118 & 9.3 \\
RHA119 & 10.1 \\
\hline
\end{tabular}

RHA, RNA human acute myeloid leukemia.

org/pub/release-75/gtf/homo_sapiens/Homo_sapiens.GRCh37.75. gtf.gz) (8) using Tophat2 (version 2.1.0; Johns Hopkins University, Baltimore, MD, USA) (9). Aligned reads (BAM files) were subsequently sorted and indexed using SAMtools (version 1.2; Wellcome Trust Sanger Institute, Cambridge, UK) (10). Cufflinks (version 2.2.1; University of Washington, Seattle, WA, USA) (11) was utilized for final transcriptome assembly (cufflink and cuffmerge function), and abundance estimation and normalization in Fragments Per Kilobase of transcript per Million mapped reads (FPKM) units (cuffnorm function).

Cell-type classification. A heatmap was generated for the key cell-type specific markers (typically used inimmunophenotyping using flow cytometry, including CD34 and CD45) based on their expression levels. For any gene, the presence of a transcript with an FPKM normalized expression value $>0$ is indicative of gene expression, while a FPKM normalized expression value of 0 indicates absence of expression. The presence and absence of the cell-type specific markers were plotted in a heatmap generated using the 'pheatmap' package (version 1.0.8) produced by the R Programming Environment (www.r-project.org).

Based on the gene expression profiles, cells that were CD34-positive, or HLA-DRA- and CD117-positive, were classified as 'putative blasts' (12).

Principal component analysis. Principal component analysis was carried out on the $\log _{2}$-transformed FPKM normalized expression values of all transcripts using the prcomp function of the R Programming Environment. 


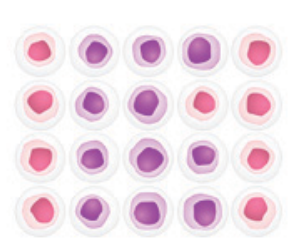

Single-cell library preparation

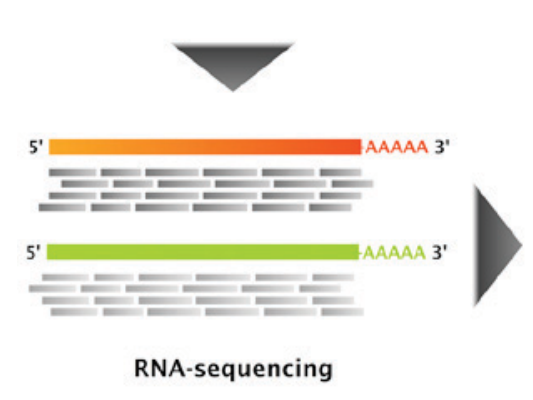

Acute myeloid leukemia bone marrow specimen

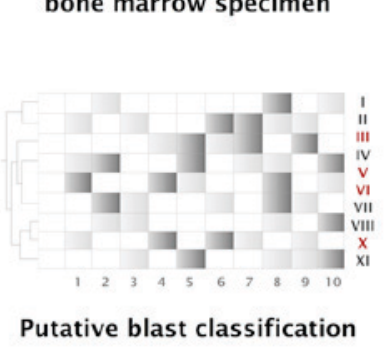

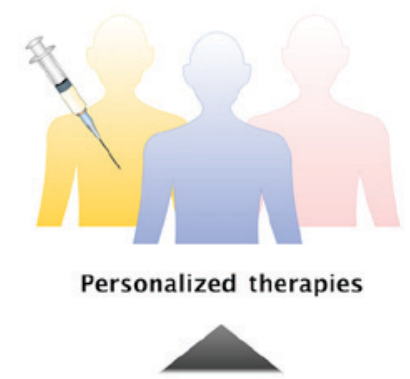

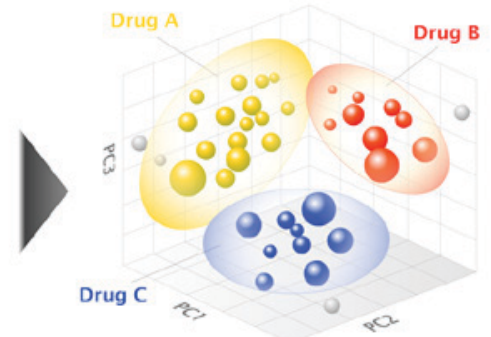

Principal component analysis of transcriptomic data

Figure 1. Scheme of a proposed clinical single-cell genomic profiling protocol. PC, principal component.

Targeted DNA-sequencing (DNA-seq). Targeted DNA-seq was performed as previously described $(13,14)$. A total of $50 \mathrm{ng}$ of genomic DNA was extracted from the AML bone marrow sample and processed using the TruSight Myeloid Sequencing Panel (Illumina, Inc.). A total of 54 genes known to be mutated inmyeloid neoplasms, including fms related tyrosine kinase 3 (FLT3), nucleophosmin (NPM1) and DNA methyltransferase 3 alpha (DNMT3A), were assessed. The TruSeq Amplicon (BaseSpace Workflow; version 1.1.0.0; Illumina, Inc.) was used to generate the BAM and VCF files. Visualization of reads was performed using the Integrative Genomics Viewer (version 2.3.69; Broad Institute, Cambridge, MA, USA) (15). Pindel (version 0.2.5a8; McDonnell Genome Institute, Washington University School of Medicine, St. Louis, MO, USA) (16) was used to identify the presence of FLT3 internal tandem duplications.

Variant calling of RNA-seq data. Variant calling analysis was carried out on the aligned paired end reads using the Genome Analysis Toolkit (Broad Institute) (version3.4.46; Haplotype Caller function) with reference to the aforementioned human $\mathrm{Hg} 19$ genome $(17,18)$. Variants identified from the analysis were annotated using the SeattleSeq Annotation webserver (snp.gs.washington. edu/SeattleSeqAnnotation138) (19). Visualization of reads was performed using the SAMtools tview function (10).

\section{Results}

Number of RNA-seq reads per cell. The number of reads per cell was between 4.5 million and 11.4 million (Table I), which is consistent with previous single-cell RNA-seq studies (20-23).

Cell-type classification. Immunophenotyping using flow cytometry demonstrated the blast population to comprise of $\sim 65 \%$ the total number of cells. Based on the single-cell gene expression profile, 11/20 cells were identified to be putative blasts (Fig. 2).

Principal component analysis. Principal component analysis was performed in an attempt to identify potential subclonal populations (Fig. 3). Two outlier cells were identified, RHA115 and RHA118. Based on their gene expression profile (Fig. 2), these cells were classified as putative blasts.

Variant calling of RNA-seq data. Targeted DNA-seq revealed the presence of aDNMT3A mutation (c.2644C>T; p.Arg882Cys; Fig. 4); an NPM1 mutation (c.859_860insTCTG; p.Trp288CysfsTer12); and a 108 bp FLT3 internal tandem duplication (data not shown).

Variant calling of the RNA-seq data did not identify cells with any of the aforementioned NPM1 and FLT3 mutations. The DNMT3A mutation (c.2644C>T; p.Arg882Cys) was identified in one cell (RNA human AML119) (Fig. 5). Coverage analysis was performed in an attempt to understand the apparent absence of NPMI and FLT3 transcript mutations, and low abundance of DNMT3A transcript mutations across the 20 cells. This revealed the reason to be the absence of transcripts mapping to the relevant mutation site, potentially secondary to stochastic transcript dropout (24).

\section{Discussion}

Single-cell genomic analysis of AML has been previously reported $(25,26)$. However, these studies involved only DNA analysis. To the best of our knowledge, the present study is the first single-cell transcriptomic analysis of AML.

In the present study, a clinical workflow for single-cell transcriptomic profiling has been piloted. Using single-cell RNA-seq, putative blasts were identified based on the gene expression profile of conventional immunophenotypic markers used in routine flow cytometry. For flow cytometric analysis, 


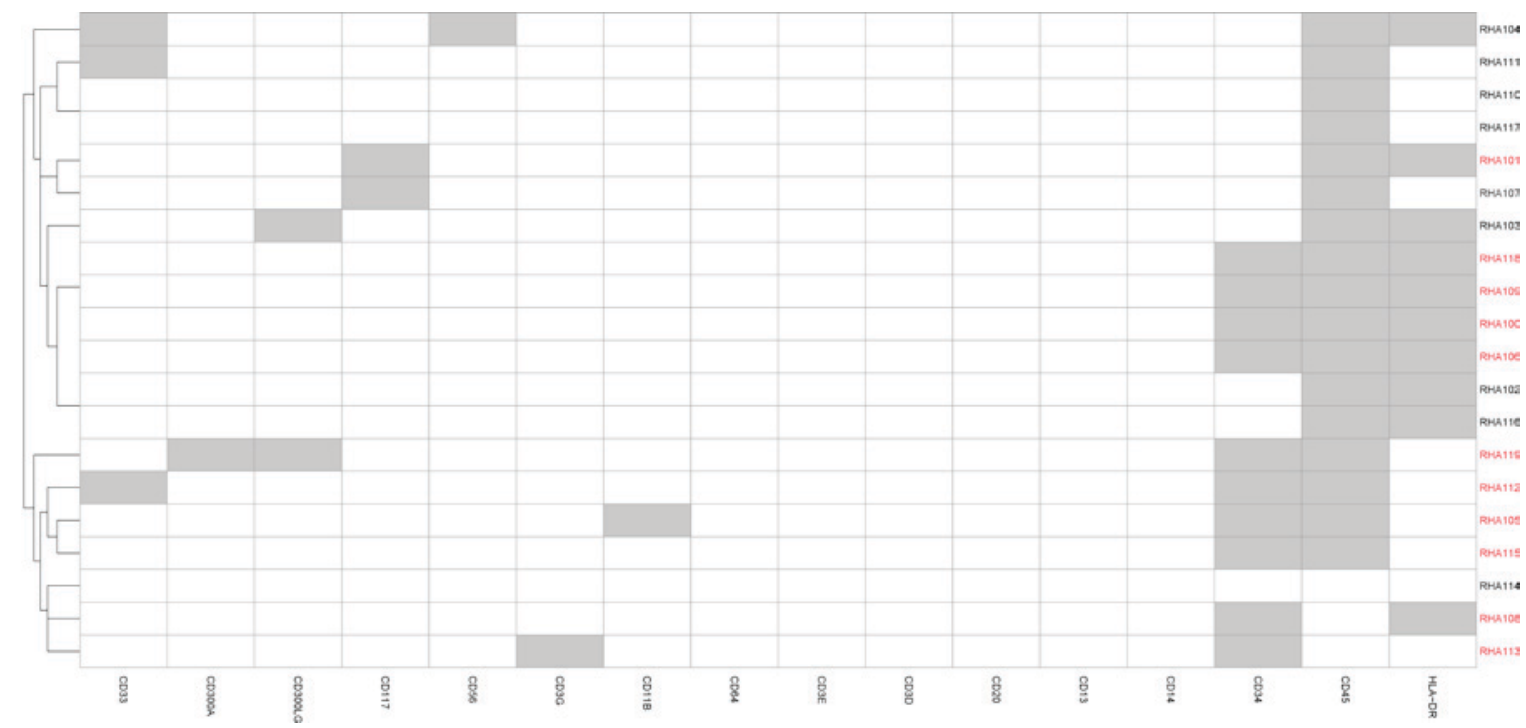

Figure 2. Single-cell gene expression profile of the 20 cells. Putative blasts are labeled red. CD, cluster of differentiation; HLA-DR, human leukocyte antigenantigen D related; RHA, RNA human acute myeloid leukemia.

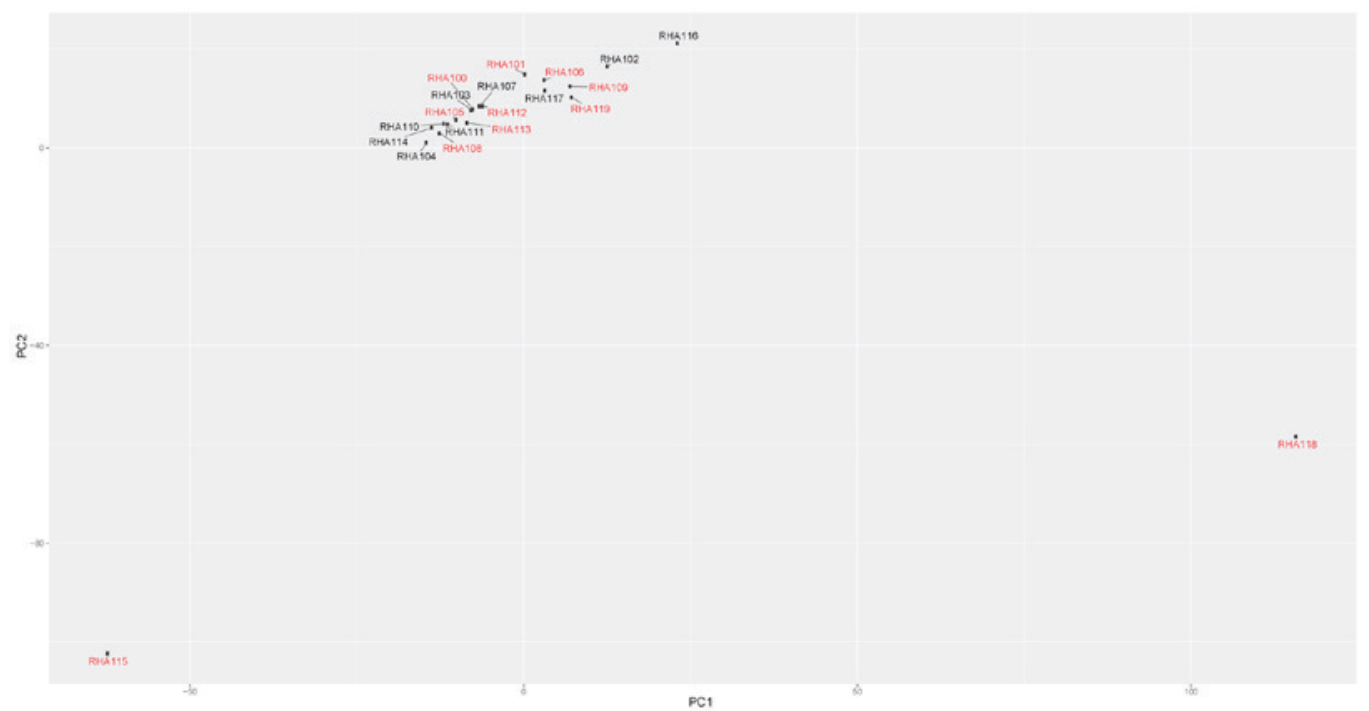

Figure 3. PC analysis of transcriptomic data. Putative blasts are labeled red. PC, principal component; RHA, RNA human acute myeloid leukemia.

20 markers are typically used for profiling. There is a large contrast with transcriptomic analysis, as there are in principle $\geq 20,000$ markers (genes) (27) that can be utilized, and individual cellular characterization is able theoretically to be highly detailed.

In addition to information derived from expression profiling, mutational (variant) data provides further information that maybe useful for individual cell categorization (26). Variant identification is most commonly performed on DNA-seq data (28). However, variant identification has also been performed on bulk (29) and single-cell RNA-seq data $(23,30)$. In the present study, the DNMT3Ap.Arg882Cys mutation was identified in the transcript, providing evidence that the mutant transcript is expressed.

High-dimensional data presents an opportunity for increased cellular characterization and the potential identification of subclonal populations. Principal component analysis of the dataset in the present study revealed two putative blasts that did not cluster with the other blasts. In future studies, the authors of the present study aim to investigate the possibility of predicting the drug sensitivity of putative subclonal populations based on high-dimensional characterization, as has been performed in previous studies (31).

One of the primary limitations of the protocol proposed in the present study is the stochastic RNA loss, in which between 60 and $90 \%$ of poly (A) RNA may be lost during sample preparation (24). In the presentstudy, FLT3 and NPM1 transcript mutations were not identified in the 20 cells, while the DNMT3A (c.2644C>T; p.Arg882Cys) transcript mutation was identified in one cell. Following further analysis, this observation maybe explained by the absence of transcripts mapping to the relevant mutation site. Significant methodological improvements and protocol optimization are required to overcome this limitation. 


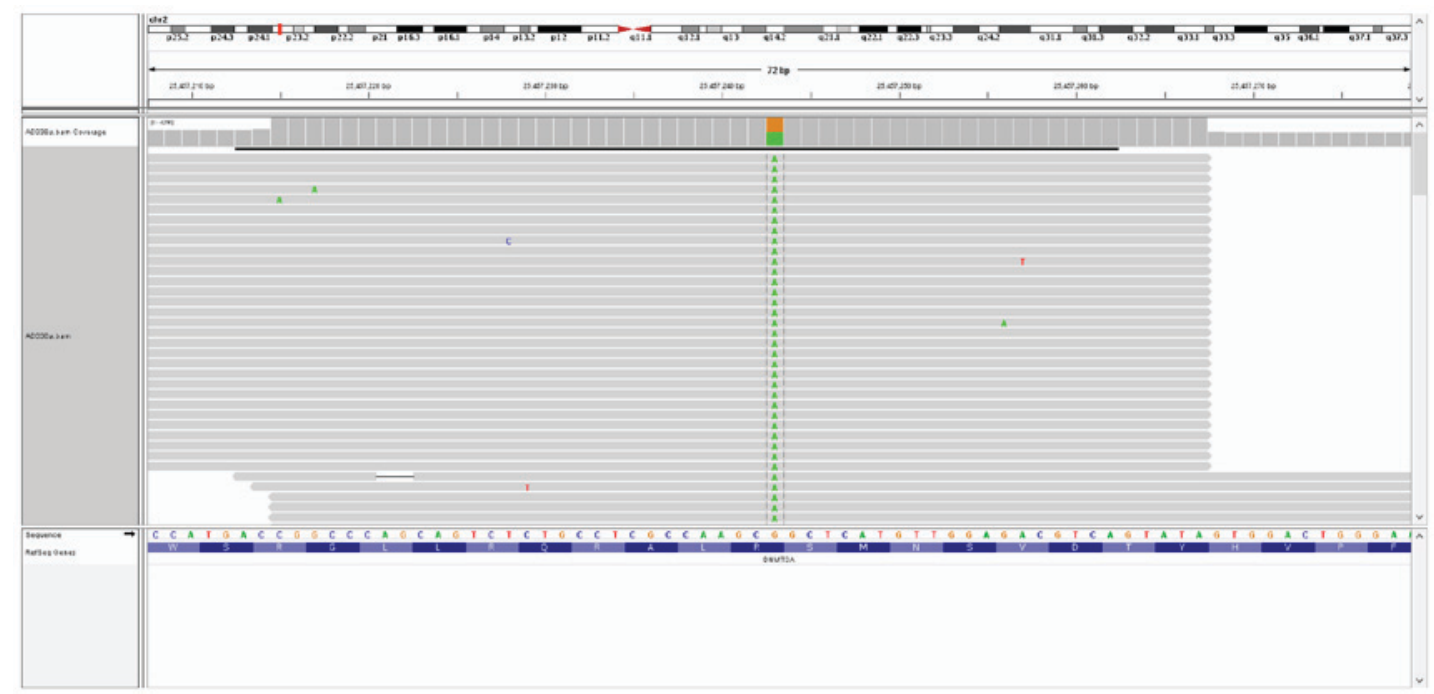

Figure 4. DNMT3Ap.Arg882Cys mutation was identified using targeted DNA-sequencing and visualized using the Integrative Genomics Viewer.DNMT3A, DNA methyltransferase 3 alpha; RefSeq, reference sequence.

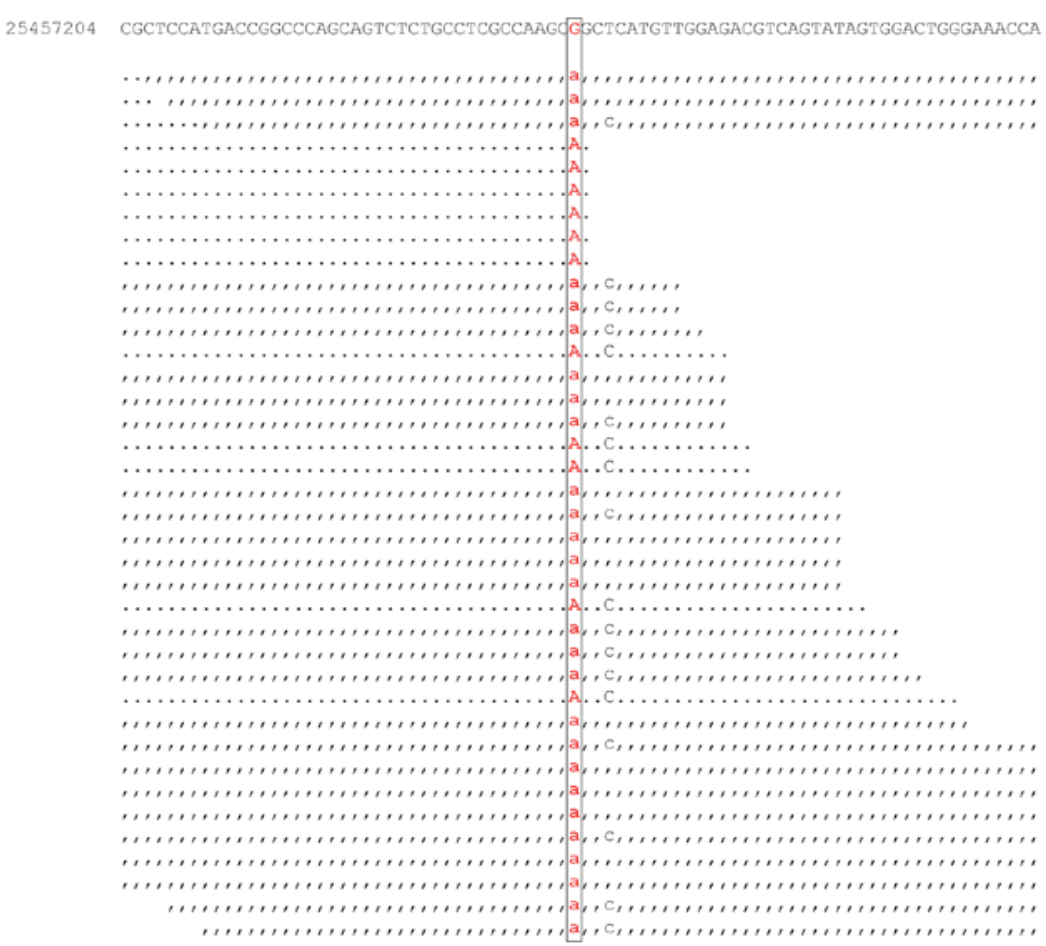

Figure 5. DNA methyltransferase 3 alpha p. Arg882Cys mutation was identified using RNA-sequencing and visualized using the SAMtools tview function.

Another current limitation is the relatively low throughput of the protocol proposed in the present study. Due to reasons of cost and logistics, routine clinical genomic profiling of a hundred cells is currently challenging (32). By contrast, flow cytometric immunophenotyping typically involves profiling tens of thousands of cells (33). With the development of higher-throughput platforms $(2,3)$, there is the potential that the cost of single-cell genomic profiling will decrease significantly to a point where it becomes viable for clinical implementation.

Despite the aforementioned limitations, single-cell genomic profiling may lead to the improved diagnosis and theragnosis of various types of cancer, including AML. In the present study, a possible single-cell genomic profiling protocol was piloted for clinical diagnostics. In future studies, a larger number of cells may need to be profiled to identify distinct subclonal populations and predict respective drug sensitivity profiles based on subclonal genomic signatures.

\section{Acknowledgements}

The present study was supported by the Clinician Scientist Award (grant no. NMRC/CSA/046/2012) and the Clinician Scientist Individual Research Grant (grant 
no. CIRG/1379/2013) from the National Medical Research Council (Singapore) awarded to R.F.

\section{References}

1. Alizadeh AA, Aranda V, Bardelli A, Blanpain C, Bock C Borowski C, Caldas C, Califano A, Doherty M, Elsner M, et al: Toward understanding and exploiting tumor heterogeneity. Nat Med 21: 846-853, 2015 .

2. Klein AM, Mazutis L, Akartuna I, Tallapragada N, Veres A, Li V, Peshkin L, Weitz DA and Kirschner MW: Droplet barcoding for single-cell transcriptomics applied to embryonic stem cells. Cell 161: 1187-1201, 2015

3. Macosko EZ, Basu A, Satija R, Nemesh J, Shekhar K, Goldman M, Tirosh I, Bialas AR, Kamitaki N, Martersteck EM, et al: Highly parallel genome-wide expression profiling of individual cells using nanoliter droplets. Cell 161: 1202-1214, 2015.

4. Herzenberg LA, Parks D, Sahaf B, Perez O, Roederer M and Herzenberg LA: The history and future of the fluorescence activated cell sorter and flow cytometry: A view from Stanford. Clin Chem 48: 1819-1827, 2002.

5. Döhner H, Weisdorf DJ and Bloomfield CD: Acute myeloid leukemia. N Engl J Med 373: 1136-1152, 2015.

6. Ding L, Tey TJ, Larson DE, Miller CA, Koboldt DC, Welch JS, Ritchey JK, Young MA, Lamprecht T, McLellan MD, et al: Clonal evolution in relapsed acute myeloid leukaemia revealed by whole-genome sequencing. Nature 481: 506-510, 2012

7. van Dongen JJ, Lhermitte L, Böttcher S, Almeida J, van der Velden VH, Flores-Montero J, Rawstron A, Asnafi V, Lécrevisse Q, Lucio P, et al: EuroFlow antibody panels for standardized n-dimensional flow cytometric immunophenotyping of normal, reactive and malignant leukocytes. Leukemia 26 : 1908-1975, 2012

8. Cunningham F, Amode MR, Barrell D, Beal K, Billis K, Brent S, Carvalho-Silva D, Clapham P, Coates G, Fitzgerald S, et al: Ensembl 2015. Nucleic acids Res 43: D662-D669, 2015.

9. Kim D, Pertea G, Trapnell C, Pimentel H, Kelley R and Salzberg SL: TopHat2: Accurate alignment of transcriptomes in the presence of insertions, deletions and gene fusions. Genome Bio 14: R36, 2013.

10. Li H, Handsaker B, Wysoker A, Fennell T, Ruan J, Homer N, Marth G, Abecasis G and Durbin R; 1000 Genome Project Data Processing Subgroup: The Sequence Alignment/Map format and SAMtools. Bioinformatics 25: 2078-2079, 2009.

11. Trapnell C, Williams BA, Pertea G, Mortazavi A, Kwan G, van Baren MJ, Salzberg SL, Wold BJ and Pachter L: Transcript assembly and quantification by RNA-Seq reveals unannotated transcripts and isoform switching during cell differentiation. Nat Biotechnol 28: 511-515, 2010

12. Craig FE and Foon KA: Flow cytometric immunophenotyping for hematologic neoplasms. Blood 111: 3941-3967, 2008.

13. Yan B, Hu Y, Ng C, Ban KH, Tan TW, Huan PT, Lee PL, Chiu L, Seah E, Ng CH, et al: Coverage analysis in a targeted amplicon-based next-generation sequencing panel for myeloid neoplasms. J Clin Pathol 69: 801-814, 2016.

14. Yan B, Ng C, Moshi G, Ban K, Lee PL, Seah E, Chiu L, Koay ES, Liu TC, $\mathrm{Ng} \mathrm{CH}$, et al: Myelodysplastic features in a patient with germline CEBPA-mutant acute myeloid leukaemia. J Clin Pathol 69: 652-654, 2016.

15. Thorvaldsdóttir H, Robinson JT and Mesirov JP: Integrative Genomics Viewer (IGV): High-performance genomics data visualization and exploration. Brief Bioinform 14: 178-192, 2013.

16. Ye K, Schulz MH, Long Q, Apweiler R and Ning Z: Pindel: A pattern growth approach to detect break points of large deletions and medium sized insertions from paired-end short reads. Bioinformatics 25: 2865-2871, 2009.
17. McKenna A, Hanna M, Banks E, Sivachenko A, Cibulskis K, Kernytsky A, Garimella K, Altshuler D, Gabriel S, Daly M and DePristo MA: The Genome Analysis Toolkit: A MapReduce framework for analyzing next-generation DNA sequencing data. Genome Res 20: 1297-1303, 2010.

18. Van der Auwera GA, Carneiro MO, Hartl C, Poplin R, Del Angel G, Levy-Moonshine A, Jordan T, Shakir K, Roazen D, Thibault J, et al: From FastQ data to high confidence variant calls: The Genome Analysis Toolkit best practices pipeline. Curr Protoc Bioinformatics 43: 11.10.1-33, 2013.

19. Ng SB, Turner EH, Robertson PD, Flygare SD, Bigham AW, Lee C, Shaffer T, Wong M, Bhattacharjee A, Eichler EE, et al: Targeted capture and massively parallel sequencing of 12 human exomes. Nature 461: 272-276, 2009.

20. Pollen AA, Nowakowski TJ, Shuga J, Wang X, Leyrat AA, Lui JH Li N, Szpankowski L, Fowler B, Chen P, et al: Low-coverage single-cell mRNA sequencing reveals cellular heterogeneity and activated signaling pathways in developing cerebral cortex. Nat Biotechnol 32: 1053-1058, 2014.

21. Shalek AK, Satija R, Shuga J, Trombetta JJ, Gennert D, Lu D, Chen P, Gertner RS, Gaublomme JT, Yosef N, et al: Single-cell RNA-seq reveals dynamic paracrine control of cellular variation. Nature 510: 363-369, 2014.

22. Treutlein B, Brownfield DG, Wu AR, Neff NF, Mantalas GL, Espinoza FH, Desai TJ, Krasnow MA and Quake SR: Reconstructing lineage hierarchies of the distal lung epithelium using single-cell RNA-seq. Nature 509: 371-375, 2014.

23. Kim KT, Lee HW, Lee HO, Kim SC, Seo YJ, Chung W, Eum HH, Nam DH, Kim J, Joo KM and Park WY: Single-cell mRNA sequencing identifies subclonal heterogeneity in anti-cancer drug responses of lung adenocarcinoma cells. Genome Biol 16: 127, 2015.

24. Kim JK, Kolodziejczyk AA, Illicic T, Teichmann SA and Marioni JC: Characterizing noise structure in single-cell RNA-seq distinguishes genuine from technical stochastic allelic expression. Na Commun 6: 8687, 2015.

25. Hughes AE, Magrini V, Demeter R, Miller CA, Fulton R, Fulton LL, Eades WC, Elliott K, Heath S, Westervelt P, et al: Clonal architecture of secondary acute myeloid leukemia defined by single-cell sequencing. PLoS Genet 10: e1004462, 2014.

26. Paguirigan AL, Smith J, Meshinchi S, Carroll M, Maley C and Radich JP: Single-cell genotyping demonstrates complex clonal diversity in acute myeloid leukemia. Sci Transl Med 7: 281re2, 2015.

27. International Human Genome Sequencing Consortium: Finishing the euchromatic sequence of the human genome. Nature 431: 931-945, 2004

28. Papaemmanuil E, Gerstung M, Bullinger L, Gaidzik VI, Paschka P, Roberts ND, Potter NE, Heuser M, Thol F, Bolli N, et al: Genomic classification and prognosis in acute myeloid leukemia. N Engl J Med 374: 2209-2221, 2016.

29. Li M, Wang IX, Li Y, Bruzel A, Richards AL, Toung JM and Cheung VG: Widespread RNA and DNA sequence differences in the human transcriptome. Science 333: 53-58, 2011.

30. Patel AP, Tirosh I, Trombetta JJ, Shalek AK, Gillespie SM, Wakimoto H, Cahill DP, Nahed BV, Curry WT, Martuza RL, et al: Single-cell RNA-seq highlights intratumoral heterogeneity in primary glioblastoma. Science 344: 1396-1401, 2014.

31. Mitra AK, Mukherjee UK, Harding T, Jang JS, Stessman H, Li Y, Abyzov A, Jen J, Kumar S, Rajkumar V and Van Ness B: Single-cell analysis of targeted transcriptome predicts drug sensitivity of single cells within human myeloma tumors. Leukemia 30: 1094-1102, 2016.

32. Shapiro E, Biezuner $\mathrm{T}$ and Linnarsson S: Single-cell sequencing-based technologies will revolutionize whole-organism science. Nat Rev Genet 14: 618-630, 2013.

33. Lee D, Grigoriadis G and Westerman D: The role of multiparametric flow cytometry in the detection of minimal residual disease in acute leukaemia. Pathology 47: 609-621, 2015. 